\section{Factores de riesgo asociados a infecciones recurrentes de tracto urinario en niños}

\author{
Risk factors associated with recurrent urinary tract infections \\ in children
}

\section{Fatores de risco associados a infecções recorrentes do trato urinário em crianças}

\author{
Jimena Rosario Vilca Yahuita \\ yimita.medped.vilca@gmail.com
}

\section{RESUMEN}

Introducción: La infección del tracto urinario (ITU) es una de las patologías infecciosas más frecuentes en pediatría. La mayor parte es causada por los anaerobios facultativos que habitualmente se originan en la flora intestinal. Objetivo: Determinar los factores de riesgo frecuentes asociados a infecciones de tracto urinario recurrente en lactantes, pre-escolares y escolares atendidos en el Hospital del Niño "Dr. Ovidio Aliaga Uría", gestión 2017. Materiales y métodos: Es un estudio de casos y controles. La población estuvo conformada por: casos, los niños de 1 mes a 10 años y 11 meses, de ambos sexos con diagnóstico de infección urinaria recurrente, con examen general de orina (EGO), urocultivo positivo y ecografía renal y de vías urinarias. El tamaño de muestra fue calculado con EPINFO para diseño de casos y controles con un nivel de confianza $95 \%$ se obtiene un tamaño de muestra para casos de 65 pacientes y 65 controles en total 130 pacientes. Para el análisis se utilizó estadística descriptiva. Resultados: Los factores asociados a ITU recurrente son las malformaciones nefro-urológicas, fimosis en el sexo masculino. No se encontró diferencia entre casos y controles en cuanto a otros factores riesgo como constipación, hábitos higiénico - dietéticos y vulvovagitis). Conclusión: El grupo etario más afectado con ITU recurrente en el presente estudio comprende a pre-escolares, con predominio del sexo femenino.

Palabras clave: Infección; urinaria; recurrencia; pediatría; riesgo
JV: Universidad Mayor de San Andrés, Bolivia.

\begin{abstract}
Introduction: Urinary tract infection (UTI) is one of the most frequent infectious pathologies in pediatrics. Most of it is caused by facultative anaerobes that usually originate in the intestinal flora. Objective: To determine the frequent risk factors associated with recurrent urinary tract infections in infants, pre-school children and school children treated at the Hospital del Niño "Dr. Ovidio Aliaga Uría", 2017 management. Materials and methods: It is a case-control study. The population consisted of: cases, children from 1 month to 10 years and 11 months, of both sexes with a diagnosis of recurrent urinary infection, with a general urine examination (EGO), positive urine culture, and renal and urinary tract ultrasound. The sample size was calculated with EPINFO for design of cases and controls with a confidence level of $95 \%$. A sample size is obtained for cases of 65 patients and 65 controls in total 130 patients. Descriptive statistics were used for the analysis. Results: The factors associated with recurrent UTI are nephro-urological malformations, phimosis in the male sex. No difference was found between cases and controls regarding other risk factors such as constipation, hygienic - dietary habits and vulvovagitis). Conclusion: The age group most affected with recurrent UTI in the present study comprises preschool children, with a predominance of women.
\end{abstract}

Key words: Infection; urinary; recurrence; pediatrics; risk 


\section{RESUMO}

JV: Universidad Mayor de San Andrés, Bolivia.
Introdução: A infecção do trato urinário (ITU) é uma das patologias infecciosas mais frequentes em pediatria. A maior parte é causada por anaeróbios facultativos que geralmente se originam na flora intestinal. Objetivo: Determinar os freqüentes fatores de risco associados a infecções recorrentes do trato urinário em lactentes, crianças em idade pré-escolar e escolares atendidos no Hospital del Niño "Dr. Ovidio Aliaga Uría", gestão de 2017. Materiais e métodos: É um estudo de caso-controle. A população foi composta por: casos, crianças de 1 mês a 10 anos e 11 meses, de ambos os sexos, com diagnóstico de infecção urinária recorrente, com exame geral de urina (EGO), cultura positiva de urina e ultrassonografia renal e do trato urinário. O tamanho da amostra foi calculado com o EPINFO para o desenho de casos e controles com um nível de confiança de $95 \%$, sendo obtido um tamanho de amostra para casos de 65 pacientes e 65 controles no total de 130 pacientes. Estatística descritiva foi utilizada para a análise. Resultados: Os fatores associados à ITU recorrente são malformações nefro-urológicas, fimose no sexo masculino. Não foi encontrada diferença entre casos e controles em relação a outros fatores de risco, como constipação, hábitos higiênico - alimentares e vulvovagite). Conclusão: A faixa etária mais acometida por ITU recorrente no presente estudo é composta por pré-escolares, com predomínio do sexo feminino.

Palavras-chave: Infecção; urinário; recorrência; pediatria; arriscar

\section{INTRODUCCIÓN}

$\mathrm{L}$ a infección del tracto urinario (ITU) es una de las patologías infecciosas más frecuentes en la infancia y se reconoce como la segunda causa de infección bacteriana (1). Se define como la invasión, colonización y proliferación bacteriana del tracto urinario, con signos y síntomas que pueden comprometer desde la vejiga hasta el parénquima renal. Los patógenos responsables en su mayoría son los anaerobios facultativos que habitualmente se originan en la flora intestinal (2).

La ITU recurrente se caracteriza por el número de episodios sintomáticos repetidos de infección urinaria (al menos 2 episodios en 6 meses o 3 episodios en 1 año) con intervalos libres de infección. Pueden ser de dos tipos: a) Recaída: debida al mismo germen a pesar del tratamiento antibacteriano adecuado dentro de las tres primeras semanas de tratamiento b) Reinfección: ITU con microorganismo distinto después de 1 a 2 semanas de tratamiento inicial (2).
Según estudios de recurrencia se estima que el $1 \%$ de los niños y $3 \%$ de las niñas presentará un episodio de ITU sintomático antes de los 11 años de edad, el riesgo es mayor durante el primer año de vida. Se calcula que las cifras de recurrencia en niños de mayor edad aumentan y guardan relación con el número de infecciones previas. La frecuencia después de una ITU inicial es de aproximadamente $30 \%$, después de la segunda y tercera infección se incrementa del 60 al $75 \%$ (1).

El diagnóstico y tratamiento precoz de la ITU ha demostrado ser determinante para evitar la aparición de cicatrices renales, por lo tanto, es importante identificar en la historia y en el examen físico factores de riesgo y elementos clínicos que sugieren una anormalidad de la vía urinaria que favorezca la primoinfección y la recurrencia de la ITU.

La ITU tradicionalmente se ha considerado como marcador de probables anormalidades anatómicas y funcionales de la vía urinaria, por lo cual, en las últimas 
décadas todos los niños que la presentan son sometidos a estudios por imágenes en busca de anormalidades de la vía urinaria o cicatrices renales (2).

Entre los factores de riesgo para la a ITU se pueden mencionar las malformaciones anatómicas asociadas o no a uropatía obstructiva, constipación, vulvovaginitis, inadecuado aseo en genital, siendo las niñas más susceptibles a las infecciones recurrentes en relación a los niños (3). Existe una amplia evidencia que las infecciones recurrentes constituyen un factor predisponente para el desarrollo de daño renal. En estudios donde se ha observado la aparición de nuevas cicatrices renales, éstas generalmente han sido precedidas por nuevos episodios de ITU (4).

La ITU afecta con mayor frecuencia a pacientes de sexo femenino en todas las edades, a excepción de los primeros 3 meses de vida, período en que predomina en los varones. Aproximadamente $3 \%$ de las niñas prepuberales y $1 \%$ de los varones de edad similar han presentado ITU (3).

El riesgo de ITU recurrente en niños se ha estimado en un $12-30 \%$ en los primeros 6 -12 meses después de la ITU inicial y generalmente son causadas por la misma cepa del episodio original. Los factores predisponentes para la recurrencia incluyen el reflujo vesicoureteral y la eliminación disfuncional (3).

En lo referente al diagnóstico microbiológico la Escherichia coli (E. coli) es el microorganismo más común que causa ITU en niños (60-92\%). Otros organismos comunes incluyen Klebsiella, Proteus, Enterococcus, Enterobacter spp, Pseudomonas, Streptococcus del Grupo B y Staphylococcus aureus (4).

El riñón joven es considerado más vulnerable a las agresiones, por lo que se ha señalado que los niños menores tienen un riesgo mayor de desarrollar daño renal después de una infección urinaria, esta podría estar asociada con una lesión renal más pronunciada debida a la morfología papilar única de esta edad.

Diversos autores han confirmado que niños pequeños tienen un mayor riesgo de desarrollar secuelas renales después de una pielonefritis aguda, mientras que otros aseguran que también los niños de más edad tienen alto riesgo de desarrollar cicatrices renales (5). En estudios donde se ha observado la aparición de nuevas cicatrices renales, se identificó que los pacientes habían presentado nuevos episodios de infecciones urinarias (6). Existe una amplia evidencia que destaca el papel de las ITU recurrentes como factor predisponente para el desarrollo de daño renal $(7,8)$.

Al ser las ITU una de las patologías bacterianas más frecuentes en la infancia con la consiguiente posibilidad de daño en el parénquima renal y potencial a generar secuelas a largo plazo, junto con el potencial de anomalías del tracto urinario se hace necesario su prevención, diagnóstico y tratamiento precoz. Está claro que esta es la clave para evitar el daño renal. El presente trabajo tiene por objetivo determinar los factores de riesgo asociados a infecciones de tracto urinario recurrente en lactantes, pre-escolares y escolares atendidos en el Hospital del Niño "Dr. Ovidio Aliaga Uría”.

\section{MATERIALES Y MÉTODO}

$\mathrm{S}$ e trata de un estudio de casos y controles, con casos incidentes de 3 meses, en el que se incluyó expedientes clínicos de todos los pacientes lactantes, pre-escolares y escolares diagnosticados con infección urinaria recurrente (casos) y episodio único (controles), se elaboró un instrumento de recolección de datos.

La población estuvo conformada por: 1) Casos, los niños de 1 mes a 10 años y 11 
meses, de ambos sexos con diagnóstico de infección urinaria recurrente, con examen general de orina (EGO), urocultivo positivo y ecografía renal y de vías urinarias. 2) Casos control, los niños de 1 mes a 10 años y 11 meses, de ambos sexos con diagnóstico de infección urinaria (1er episodio, con examen general de orina (EGO), urocultivo positivo y ecografía renal y de vías urinarias.

El tamaño de muestra fue calculado con EPINFO para diseño de casos y controles con un nivel de confianza 95\%y un poder del $80 \%$, relación caso y control $1: 1$, con un OR aproximado de 3 se obtiene un tamaño de muestra para casos de 65 pacientes y 65 controles dando un total de muestras de 130 pacientes lactantes, preescolares y escolares.

Los datos fueron ingresados en una base de datos de formato Epi-Info. La validación del ingreso de datos se realizó mediante doble entrada para la corrección de los errores de ingreso y/o registro

El análisis exploratorio se efectuó con la descripción de los datos recogidos durante el proceso de investigación a través del uso de la estadística descriptiva, un análisis univariado para la identificación de los factores de riesgo asociados a ITU recurrente en lactantes, prescolares y escolares. La fuerza de la asociación entre infección urinaria recurrente y los factores de riesgo, se midió con el Odds Radio (OR) ajustado y los intervalos de confianza al 95\%, obtenidos mediante el modelo de regresión logística condicional.

Las madres recibieron información acerca de los alcances de la investigación para solicitar su asentimiento, se les explicó el carácter confidencial y la posibilidad de rechazar la participación, por tanto, era voluntaria. La investigación fue presentada $\mathrm{y}$ aprobada en sus diferentes etapas por el Comité de Ética

\section{RESULTADOS Y DISCUSIÓN}

$\mathrm{E}$ $n$ cuanto a los casos, el $77 \%$ fueron de sexo femenino en relación al $23 \%$ que corresponde a sexo masculino. El grupo etario más afectado son los pre-escolares con el $40 \%$ del total. Por otro lado, el 94, $7 \%$ de los casos presentaron urocultivo positivo cuyo germen más frecuente fue E. coli dentro de otros agentes patógenos causantes de ITU recurrente se encontraron: Pseudomonas en un $3 \% \quad \mathrm{y}$ Enterocococo 1. 8\%.

Tabla 1. Factores de riesgo asociados a infecciones de tracto urinario recurrente en lactantes, pre-escolares y escolares. Datos demográficos

\begin{tabular}{lll}
\hline PACIENTES & FA & PORCENTAJE (\%) \\
\hline EDAD & & \\
1 mes a 2 años & 17 & 26 \\
$2-5$ años & 26 & 40 \\
5 a 10 años & 22 & 34 \\
SEXO & & 23 \\
Masculino & 15 & 77 \\
Femenino & 50 & \\
GERMEN AISLADO & & 94,7 \\
E. coli & 54 & 3,5 \\
Pseudomona & 2 & 1,8 \\
Enterococcus & 1 &
\end{tabular}

Fuente. Elaboración propia 
Los factores asociados a ITU recurrente encontrados en el presente estudio, fueron las malformaciones congénitas y fimosis. En relación a las malformaciones congénitas que presentan los pacientes, incrementa el riesgo en 8.5 veces más para desarrollar ITU recurrente comparada con los que solo presentaron un episodio de ITU, es decir los controles OR 9.5 (IC 95\% 2,6 - 34.6).

La fimosis fue el segundo factor de riesgo asociado a ITU recurrente en la población de estudio, que incrementa el riesgo en 2,1 veces más en relación a pacientes control OR 3.1 (IC 95\% 0.3130.7)

Tabla 2. Factores de riesgo asociados a infecciones recurrentes de tracto urinario en lactantes, pre-escolares y escolares

\begin{tabular}{|c|c|c|c|c|c|c|}
\hline \multirow[t]{2}{*}{ FACTOR DE RIESGO } & \multicolumn{2}{|c|}{$\begin{array}{l}\text { CASOS } \\
n=65\end{array}$} & \multicolumn{2}{|c|}{$\begin{array}{l}\text { CONTROLES } \\
\mathrm{n}=65\end{array}$} & \multirow[t]{2}{*}{ OR } & \multirow[t]{2}{*}{ IC - 95\% } \\
\hline & $\mathrm{N}$ & $\%$ & $\mathrm{~N}$ & $\%$ & & \\
\hline Malformaciones congénitas & 22 & 88 & 3 & 12 & 9.5 & $2.6-34.6$ \\
\hline Constipación & 37 & 55.2 & 30 & 44,7 & 1.54 & $0,77-3.0$ \\
\hline Hábitos higiénico dietéticos & 30 & 47.62 & 33 & 52.38 & 0.81 & $0.39-1.67$ \\
\hline Fimosis & 3 & 75.0 & 1 & 25.0 & 3.1 & $0.31-30.7$ \\
\hline Vulvovaginitis & 8 & 50,0 & 8 & 50,0 & 1.01 & $0.35-2.91$ \\
\hline
\end{tabular}

Fuente: Elaboración propia

En relación a las manifestaciones clínicas en ambos grupos, la fiebre fue el signo de mayor presentación con $44 \%$ seguido de la disuria y orinas fétidas.

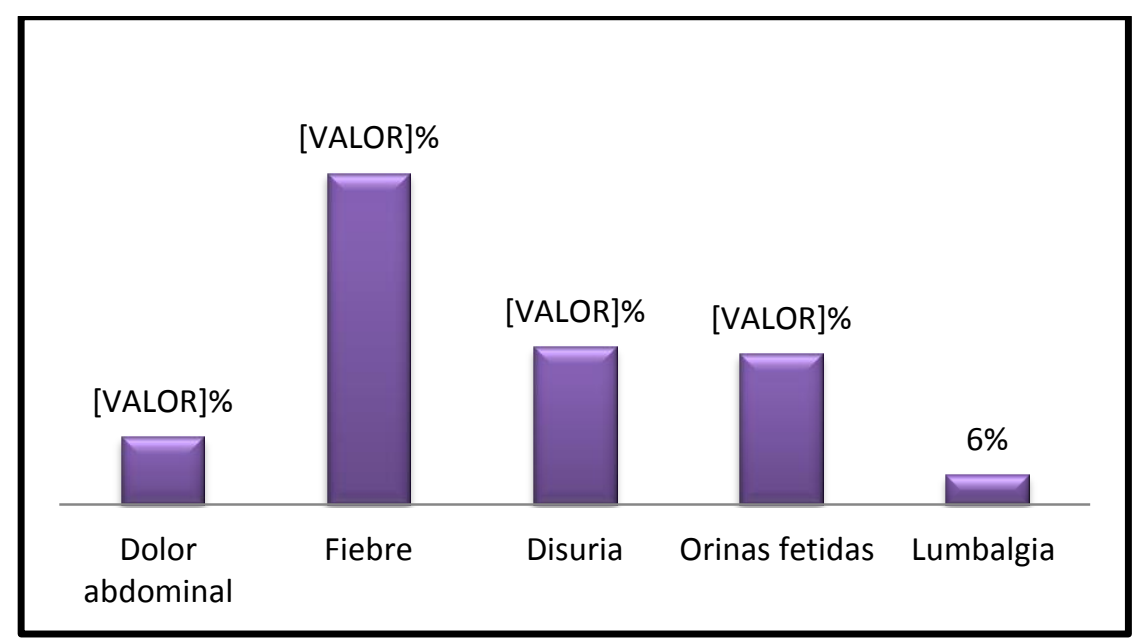

Gráfico 1. Manifestaciones clínicas en pacientes pediátricos con infecciones recurrentes de tracto urinario.

El grupo etario más afectado con ITU recurrente en el presente estudio comprende a pre-escolares, con predominio del sexo femenino, al igual que los hallazgos mencionados en la literatura
(3), esto se explica en base a la estructura anatómica, la menor longitud de la uretra femenina, lo cual contribuye a colonización, agregándose a ello la agresividad del patógeno. Según 
Valdevenito (9) las mujeres con ITU-R tienen una mayor predisposición a la colonización vaginal por uropatógenos, debido a una mayor propensión de las bacterias a adherirse a las células epiteliales. Las bacterias (E. coli) se fijan con mayor avidez a las células uroepiteliales de las niñas o mujeres susceptibles a esta patología, lo que explica el predominio de este proceso infeccioso en el sexo femenino (9).

En un estudio de revisión de casos de Chile, destaca la frecuencia de afección de dicha patología en el sexo femenino en todas las edades (Pre- escolares y escolares), a excepción de los primeros meses de vida (Lactante) que predomina en varones, cuyos datos son similares al estudio (10).

La E. coli representó el mayor agente causante de ITU recurrente, coincidiendo con lo descrito en poblaciones similares, evaluadas en otros estudios (11). En otro estudio Lozano et al (12) también identificaron la presencia de esta bacteria en 64 pacientes pediátricos con ITU, al igual que López (13) y Castaño et al (14) también encontró como principal agente a E. coli.

La manifestación clínica más destacada en el estudio fue la fiebre, la misma respaldada por la literatura (15), que sugiere de inicio descartar una asociación con cuadros gastrointestinales o respiratorios, siendo la primera opción posterior la afección de vías urinarias, sin embargo, este signo es inespecífico para realizar diagnóstico clínico.

Existe también una amplia evidencia de que las infecciones recurrentes constituyen un factor predisponente para el desarrollo de daño renal (8).

En relación a estudios sobre factores asociados para desarrollar ITU recurrente son muy pocos, y más escasos los de forma prospectiva, debido a lo anterior, se realizó un estudio prospectivo en un grupo de pacientes pediátricos, cuyo objetivo fue identificar factores de riesgo descritos en la literatura. Los factores de riesgo para ITU, identificados solo por interrogatorio y examen físico, fueron más frecuentes en el sexo femenino. Destacan el mal aseo genital, estreñimiento, el antecedente de ITU previa y la malformación de vías urinarias (11).

Las anomalías estructurales, más frecuentemente encontradas en niños que han tenido una ITU son dilataciones de la vía urinaria y riñón con doble sistema pielocalicial (16). En el presente estudio se encontró las malformaciones nefrourológicas (Hidronefrosis y patologías obstructivas) como factor principal asociado a ITU recurrente, seguida de la fimosis en el sexo masculino. No destaca la constipación, vulvovaginitis ni hábitos higiénico-dietéticos como factores principales de ITU recurrente ya que su prevalencia es elevada en ambos grupos de estudios (casos y controles), considerando que son factores modificables.

El antibiótico identificado de mayor uso tras el diagnóstico clínico y exámenes complementarios, fue el grupo de cefalosporinas de 3ra generación (cefotaxima ${ }^{\circledR}$ ) los primeros tres días, seguida de cefalosporina de segunda generación (cefixime®) los siguientes 5 días para completar esquema de tratamiento, con control de urocultivo negativo. Por otro lado, un número importante de pacientes recibieron nitrofurantoina como profilaxis nocturna, todos ellos contaban con alguna malformación urológica. Además, se encontraron algunos casos de pacientes que recibieron tratamiento previo con aminoglucósidos, cotrimoxazol sin mejoría clínica.

Según otras revisiones el antibiótico de elección al igual que en este estudio son las cefalosporinas de 3ra generación, 
seguida de aminoglucósidos, pero no mayor a 72 horas por su nefrotoxicidad (17).

Se ha identificado la resistencia de antibióticos como un posible factor de ITU recurrente (18), por el contrario, en los urocultivos reportados en el presente estudio la sensibilidad coincide con el tratamiento previamente indicado por lo que no se incluye en los factores de riesgo asociado a ITU recurrente.

\section{CONCLUSIÓN}

$\mathrm{L}$ a ITU recurrente es una de las causas más importantes de bacteriemia en niños, el grupo etario más afectado son los Pre-escolares y el sexo femenino. Los factores asociados a ITU recurrente fueron las malformaciones nefro-urológicas (Hidronefrosis patologías obstructivas), además de un importante número de pacientes con fimosis. No se encontró marcada diferencia entre casos y controles en cuanto a otros factores riesgo como constipación, hábitos higiénicos, dietéticos y vulvovagitis.

- Conflicto de intereses. Ninguno declarado por los autores.

- Financiación. Ninguna declarada por los autores

- Agradecimiento: Ninguno manifestado por los autores

- Investigación realizada considerando los tratados bioéticos

\section{REFERENCIAS BIBLIOGRÁFICAS}

1. Cueto M. La microbiología en el diagnóstico de la infección del tracto urinario. Savat; Madrid.2012. Disponible en: https://www.seimc.org/contenidos/d ocumentoscientificos/otrosdeinteres/ seimc-dc2013-

LibroInfecciondeltractoUrinario.pdf

2. Lahoud A.V, Rampoldi L.O, Saldaña F.L, Gutiérrez R.C, Susanibar N.J. Infección del Tracto Urinario
Recurrente en Pediatría. Odontología Sabnarquinba, 2001;(1):25-28

3. Pavanello R, Silva C, Frota S, Romero C, Soares A et al. Principales factores de riesgo de infección del tracto urinario (ITU) en pacientes hospitalizados: propuesta de mejoras. Enferm. Glob. [Internet]. 2009 [citado 15 junio 2016]; Disponible en: http://scielo.isciii.es/scielo.php?script =sci_arttext\&pid=S1695-

4. Orellana P, Cavagnaro F. Factores de riesgo de daño renal permanente en niños con infección del tracto urinario. Rev. Méd. Chile [Internet]. 2002 [citado enero 2017]; 130, (10): 11471153.doi.org/10.4067/S003498872002001000009

5. Coulthard MG, Lambert HJ, Vernon SJ, Hunter EW, Keir MJ, Matthews JN. Does prompt treatment of urinary tract infection in preschool children prevent renal scarring: mixed retrospective and prospective audits. Arch Dis Child. [Internet]. Consultado enero 2017. 2014; 99, (4):342-7. http://doi.org/gcbmqh

6. González J., Méndez P., Urinary tract infection in the childhood and image tests: towards a new model. Galicia Clin. [Internet]. Consultado enero 2018. 2017; 70, (3): 13-24

7. Salas C, Barrera B, González C, Zambrano O, Salgado D, Quiroz L. Actualización en el diagnóstico y manejo de la Infección Urinaria en pediatría. Rev Chil Pediatr. [Internet]. [Citado marzo 2017].2012; 83 (3): 269-78. doi.org/10.4067/S037041062012000300009

8. Pérez L, Durán D, Marchena J, Pérez Y, Rodríguez Y, Florín J. Cicatriz renal: factores de riesgo relacionados con infección urinaria. Rev Cubana Pediatr [Internet].

2007 [citado febrero 2016]; 79(2). Disponible en: http://scielo.sld.cu/scielo.php?script= sci_arttext\&pid=S0034$75312007000200004 \& \operatorname{lng}=\mathrm{es}$

9. Valdevenito J. Infección urinaria recurrente en la mujer. Rev. Chil. Infectol. [Internet]. 2008 [citado marzo 2017]; 25(4): 268-276. 
http://dx.doi.org/10.4067/S071610182008000400004

10. Cavagnaro F. Infección urinaria en la infancia. Revista Chilena de infectología. [Internet]. [Citado abril 2017] 2005; 22(2):161-68

11. Pérez M, Duran C., Marchena B, Pérez Y, Rodríguez Y, Florín Y. Cicatriz renal: factores de riesgo relacionados con infección urinaria. Rev. Cubana Pediatr. [Internet]. [Citado marzo 2017] 2007; 79 (2)

12. Lozano JM, Domínguez MM, Marrugo T. Hallazgos paraclínicos y microbiológicos en infección urinaria en pediatría en el Hospital Universitario de San Ignacio. Universitas Médica. [Internet]. [Citado marzo 2017]; 2000; 41(4):194-199

13. López JJ. Infección urinaria en pediatría. Evaluación del antibiótico empírico inicial. Rev. Fac. Med. [Internet]. [Citado marzo 2018. 2017; 42(2):51-62.

http://dx.doi.org/10.15446/revfacme d.v66n3.5997814.-14.-

14. Castaño I, González C, Buitrago Z, De Rovetto C. Etiología y sensibilidad bacteriana en infección urinaria en niños. Hospital Infantil Club Noel y Hospital Universitario del Valle, Cali, Colombia. Colombia Médica. [Internet]. [Citado marzo 2017]; 2007; 38,(2):100-106. Disponible en: http://www.scielo.org.co/pdf/cm/v3 8n2/v38n2a01.pdf
15. Hoyos A, Serna L, Aterhortúa P, Ortiz G, Aguirre J. Infección urinaria de la comunidad en pacientes pediátricos de la Clínica Universitaria Bolivariana. Etiología, presentación clínica, factores de riesgo y respuesta clínica a la terapia empírica inicial. MEDICINA UPB. [Internet]. [Citado febrero 2017]; 2010; 29 (2):89-98

16. Puñales I, Monzote A, Torres G, Hernández E. Etiología bacteriana de la infección urinaria en niños. Rev Cubana Med Gen Integr [Internet]. [Citado enero 2017]; 2012; 28(4): 620-629. Disponible en: http://scielo.sld.cu/scielo.php?script= sci_arttext\&pid=S0864-

21252012000400006\&lng=es

17. Lloyd J, Horik C, Benjamin D, Clark R, Routh J, Smith B. Incidence of Breakthrough Urinay Tract Infecction in Hospitalized Infants.ReceivingAntibioticProphylaxi s.ClinicalPediatrics. [Internet]. [Citado Enero 2017] 2017;(56):56-70. https://doi.org/10.1016/j.jpurol.2017 .08 .018

18. Echevarría-Zarate J, Sarmiento E, Osores-Plenge F. Infección del tracto urinario y manejo antibiótico. Acta méd. Peruana [Internet]. [Citado 2017 Ene 07]; 2006; 23(1): 26-31. Disponible en: http://www.scielo.org.pe/scielo.php? script $=$ sci_arttext\&pid=S172859172006000100006\&lng=es 